\title{
TRACING THE SOURCES OF COSMIC RAYS WITH MOLECULAR IONS
}

\author{
Julia K. Becker ${ }^{1}$, John H. Black ${ }^{2}$, Mohammadtaher SafarzadeH ${ }^{2,3}$, and Florian Schuppan ${ }^{1}$ \\ ${ }^{1}$ Ruhr-Universität Bochum, Fakultät für Physik \& Astronomie, Theoretische Physik IV, D-44780 Bochum, Germany; julia@tp4.rub.de \\ ${ }^{2}$ Department of Earth and Space Sciences, Chalmers University of Technology, Onsala Space Observatory, SE-439 92 Onsala, Sweden \\ Received 2011 June 23; accepted 2011 July 21; published 2011 September 6
}

\begin{abstract}
The rate of ionization by cosmic rays (CRs) in interstellar gas directly associated with $\gamma$-ray-emitting supernova remnants (SNRs) is for the first time calculated to be several orders of magnitude larger than the Galactic average. Analysis of ionization-induced chemistry yields the first quantitative prediction of the astrophysical $\mathrm{H}_{2}^{+}$emission line spectrum, which should be detectable together with $\mathrm{H}_{3}^{+}$lines. The predicted coincident observation of those emission lines and $\gamma$-rays will help prove that SNRs are sources of CRs.
\end{abstract}

Key words: acceleration of particles - cosmic rays - radiation mechanisms: non-thermal - radio continuum: general - shock waves - stars: winds, outflows - supernovae: general

Online-only material: color figures

\section{INTRODUCTION}

One of the central questions in modern physics concerns the origin of cosmic rays (CRs). The CRs themselves do not directly reveal their sources as they interact with cosmic magnetic fields and particles on their way through the universe (Strong et al. 2007). One method of identifying CR sources indirectly is the search for high-energy photon or neutrino signals. When protons of energy $E>200 \mathrm{MeV}$ interact with photon or matter fields in the vicinity of the $\mathrm{CR}$ production site, charged and neutral pions are produced, which then emit high-energy neutrinos and photons. Those neutral particles can be used to trace CRs, as they point back to the sources. This approach is limited by the low interaction cross sections that make neutrinos difficult to detect (Becker 2008), and the competition with other processes (inverse Compton scattering, bremsstrahlung) also producing photons (Schlickeiser 2002). It is therefore crucial to use multi-wavelength approaches to identify CR sources through correlation studies, and not to rely solely on the observation of high-energy photons.

The illumination of molecular clouds (MCs) by CRs from supernova remnants (SNRs) was first suggested in the 1970s by Black \& Fazio (1973) and Montmerle (1979). Most recently, observations performed with the Fermi telescope have revealed high-energy emission from several regions associated with an SNR located in close proximity to MCs. In particular, emission at $0.2-200 \mathrm{GeV}$ has been detected toward the SNRs W51C (Abdo et al. 2009), W28 (Abdo et al. 2010a), W44 (Abdo et al. 2010b), W49B (Abdo et al. 2010c), and IC443 (Abdo et al. 2010d). SNRs are one of the primary candidates for CR acceleration, as discussed for example by Biermann et al. (2009). The observed gamma-ray signatures could therefore be interpreted as $\pi^{0}$-decay products from high-energy CR interactions with the MC. The data for these particular objects seem to favor a hadronic scenario over a leptonic one (see, e.g., Abdo et al. 2010b). Due to the ambiguity of the signal, however, other tracers of CRs are needed to have a final proof.

\footnotetext{
3 Now at the Department of Physics and Astronomy, Johns Hopkins University, 3400 N. Charles Street, Baltimore, MD 21218, USA.
}

In this Letter, we quantify a new method for identifying SNRs as CR sources, based on CR-induced ionization. When CRs are accelerated in an SNR and then injected into a nearby MC, the ionization rate in the ambient gas is expected to be enhanced compared with the ionization caused by the general background of Galactic CRs. The immediate products are hydrogen ions, $\mathrm{H}^{+}$and $\mathrm{H}_{2}^{+}$, which react rapidly with $\mathrm{H}_{2}$ and oxygen to form molecular ions. Ions like $\mathrm{H}_{3}^{+}, \mathrm{OH}^{+}, \mathrm{H}_{2} \mathrm{O}^{+}$, and $\mathrm{H}_{3} \mathrm{O}^{+}$are spectroscopically observable at infrared and submillimeter wavelengths and their abundances are predictable as functions of the ionization rate (e.g., Black 1998; Montmerle 2010; and references therein). Among the 18 molecular cations now identified in the interstellar medium (ISM), it is the reactive ions $\mathrm{H}_{3}^{+}, \mathrm{OH}^{+}$, and $\mathrm{H}_{2} \mathrm{O}^{+}$that are expected to be the most specific tracers of the CR ionization rate (Black 1998, 2007), and it was only during the last year that the latter two species have been detected (Benz et al. 2010; Bruderer et al. 2010; Gerin et al. 2010; Gupta et al. 2010; Krełowski et al. 2010; Neufeld et al. 2010; Ossenkopf et al. 2010; Schilke et al. 2010; Wyrowski et al. 2010). Analysis of $\mathrm{OH}^{+}$and $\mathrm{H}_{2} \mathrm{O}^{+}$absorption in diffuse interstellar gas of low molecular fraction $\left(\mathrm{H}_{2} / \mathrm{H}<10 \%\right)$ serves to calibrate the background ionizing frequency of interstellar hydrogen at a level $\zeta_{\text {Gal }}=(0.6-2.4) \times 10^{-16} \mathrm{~s}^{-1}$ per atom (Gerin et al. 2010; Neufeld et al. 2010).

\section{IONIZATION IN THE VICINITY OF SUPERNOVA REMNANTS}

In this Letter, we estimate the enhanced interstellar ionization rates expected from the observed fluxes of high-energy gamma rays toward SNRs and predict the abundances of reactive molecular ions to be expected in associated MCs. In this way, we show how correlations between $\mathrm{GeV}-\mathrm{TeV}$ emission and vibrational and rotational spectra of molecular ions could be used to identify the sites of CR acceleration. Observational tests will be possible through astronomical spectroscopy at infrared and millimeter/submillimeter wavelengths.CRs of kinetic energies between $E_{p} \sim 1 \mathrm{MeV}$ and $\sim 1 \mathrm{GeV}$ ionize the ISM, while those at lower energy hardly escape their sources. Here, we investigate five SNRs located in or close to MCs, which are detected in gamma rays. 
Table 1

Parameters Used to Derive the Primary CR Spectrum

\begin{tabular}{lccccc}
\hline \hline Sources & W51C & W44 & W28 & IC443 & W49B \\
\hline$s$ & 1.5 & 1.74 & 1.7 & 2.09 & 2.0 \\
\hline$\Delta s$ & 1.4 & 1.96 & 1.0 & 0.78 & 1.4 \\
\hline$p_{\text {br }}\left(\mathrm{GeV} c^{-1}\right)$ & 15 & 9 & 2 & 69 & 4 \\
\hline$W_{p}\left(10^{49} \mathrm{erg}\right)$ & 9.0 & 12.1 & 3.3 & 28.0 & 35.0 \\
\hline$V\left(\mathrm{~cm}^{3}\right)$ & $3.3 \times 10^{60}$ & $4.2 \times 10^{59}$ & $3.2 \times 10^{59}$ & $4.2 \times 10^{59}$ & $6.3 \times 10^{56}$ \\
\hline
\end{tabular}

\subsection{Primary Particle Spectra}

The primary CR spectra can be deduced from the measured gamma-ray flux at Earth:

$$
\frac{d N_{\gamma}}{d E_{\gamma} d t d A_{\text {Earth }}}
$$

Assuming that the photons arise from hadronic interactions between the SNR and the MC, a primary CR spectrum can be derived. At the source, we assume a smoothed broken power law for the primaries:

$$
\frac{d N_{p}}{d E_{p} d t d A_{\text {source }}}=a_{p}\left(\frac{p}{1 \mathrm{GeV} c^{-1}}\right)^{-s}\left(1+\left(\frac{p}{p_{\mathrm{br}}}\right)\right)^{-\Delta s}
$$

where $a_{p}$ is the normalization of the primary spectrum and $p_{\mathrm{br}}$ is the location of the spectral break. For all sources, this shape of the primary spectrum reproduces the shape of the observed gamma-ray spectrum. Our results follow in general the original calculations by Fermi (Abdo et al. 2009, 2010a, 2010b, 2010c, 2010d) with slight modification of the smoothing of the power law, which only gives minor changes to the result.

We use the parameterization of the high-energy CR interaction model as presented in Kamae et al. (2006) in order to derive the primary spectrum from the gamma-ray observation. We use a fixed value of the hydrogen density, $n_{\mathrm{H}}=100 \mathrm{~cm}^{-3}$, and determine the CR energy $W_{p}$ needed to provide the observed gamma-ray flux. The volume of the emitting region $V$ enters the normalization of the proton spectrum. It can be derived from gamma observations and is taken from the Fermi publications. The spectral parameters $s, \Delta s$, and $p_{\text {br }}$, the total CR energy budget contributing to the interactions, $W_{p}$, and the interacting volume $V$ are listed in Table 1.

\subsection{Ionization}

To calculate the ionization rate, we use the parameterization by Padovani et al. (2009), including ionization by CR electrons, $\mathrm{CR}$ protons, and electron capture by CR protons. Only the direct ionization by primary protons turns out to be significant, the other contributions being at least one order of magnitude smaller. The ionization rate of $\mathrm{H}_{2}$ by primary protons reduces to

$$
\zeta^{\mathrm{H}_{2}}=\int_{E_{\min }}^{E_{\max }} \frac{d N_{p}}{d E_{p}} \sigma_{p}^{\mathrm{ion}}\left(E_{p}\right) d E_{p}
$$

with $d N_{p} / d E_{p}$ as the CR spectrum at a given kinetic energy $E_{p}$. The upper integration limit is set to $E_{\max }=1 \mathrm{GeV}$, as the rapidly decreasing cross section makes contributions at higher energies negligible.

Two main factors affect the ionization rate.
1. The $\mathrm{CR}$ spectrum at Earth as measured below $\sim 1 \mathrm{GeV}$ is modified from the spectrum at the source by transport effects of the ISM and the solar environment. The particle spectrum at the source can be derived from gamma-ray data up to approximately $1 \mathrm{GeV}$, but it must be estimated theoretically at lower energies. The extrapolation of the spectrum toward lower energies is one possibility. However, low-energy particles might follow a different acceleration scheme and produce a flatter or curved spectrum, see Blasi et al. (2005). The modeling of Blasi et al. (2005) was refined by Zirakashvili \& Ptuskin (2008) and Ellison \& Bykov (2011), who find a narrow spectrum centered at the maximum $\mathrm{CR}$ energy. In this scenario, low-energy CRs might be too rare to contribute significant ionization. However, the modeling in Zirakashvili \& Ptuskin (2008) and Ellison \& Bykov (2011) was done for young SNRs (ages $\lesssim 10^{3} \mathrm{yr}$ ), while the older remnants considered here (ages $>10^{4} \mathrm{yr}$ ) have a lower maximum energy, possibly allowing for the observed broken power-law spectrum even below $\mathrm{GeV}$ energies.

2. The choice of low-energy threshold $E_{\min }$ is related to propagation effects. The range of a $1 \mathrm{MeV}$ proton in hydrogen is $8.5 \times 10^{-4} \mathrm{~g} \mathrm{~cm}^{-2}$, which corresponds to a projected column density of $5.1 \times 10^{20} \mathrm{H} \mathrm{cm}^{-2}$. This is easily able to support detectable abundances of $\mathrm{OH}^{+}$ and $\mathrm{H}_{2} \mathrm{O}^{+}$in the diffuse molecular gas of the Galaxy. In Indriolo et al. (2009), a threshold of $E_{\min }=2 \mathrm{MeV}$ is recommended for diffuse clouds with hydrogen number densities $n_{\mathrm{H}}<10^{3} \mathrm{~cm}^{-3}$, and $E_{\min }=10 \mathrm{MeV}$ for dense clouds, where $n_{\mathrm{H}}>10^{3} \mathrm{~cm}^{-3}$. Close to a CR accelerator, at an average density of $100 \mathrm{~cm}^{-3}$, values above $2 \mathrm{MeV}$ are reasonable choices to account for the stopping range.

The following approach is chosen to account for these uncertainties: we first assume that the spectrum continues as a power law down to $\mathrm{MeV}$ energies. We then introduce an artificial and sharp cutoff in the spectrum at a minimum kinetic energy of $E_{\text {min }}$, below which the CR flux is set to zero. We then vary the minimum energy between $E_{\min }=1 \mathrm{MeV}$ and $100 \mathrm{MeV}$, the first being the most optimistic scenario, the second a rather conservative approach. As the CR ionization cross section decreases with energy, increasing the threshold will remove much of the potential signal, having an even more extreme effect than flattening the CR spectrum. Increasing the threshold at the same time accounts for considering different stopping ranges. At this stage, due to the lack of knowledge of the lower part of the spectrum, we will calculate the possible range of ionization intensity rather than one specific value.

Figure 1 shows the ionization rate, normalized to the Galactic average, versus the minimum energy. For comparison, the stopping range is shown as a function of $E_{\min }$. For the most optimistic scenario, still compatible with current knowledge, $E_{\min }=2 \mathrm{MeV}$, ionization rates range from 110 to $4 \times 10^{7}$ times the Galactic average. An energy cutoff at $100 \mathrm{MeV}$ leaves W49B as an outstanding source with a factor of $4 \times 10^{4}$ above the Galactic average. The central reason is the large CR energy density in this source (Abdo et al. 2010c). At $E_{\min }=100 \mathrm{MeV}$, only W51C drops to values below $\zeta_{\mathrm{Gal}}$. All other sources remain a factor of a few to ten above the background.

Additional ionization by energetic secondary electrons that are produced in the primary ionizing event is not included. These secondary ionizations increase the total rate by factors of a few (Padovani et al. 2009), but the details are sensitive to the relative abundances of $\mathrm{H}, \mathrm{H}_{2}, e^{-}$, and $\mathrm{He}$ (Dalgarno et al. 1999). 


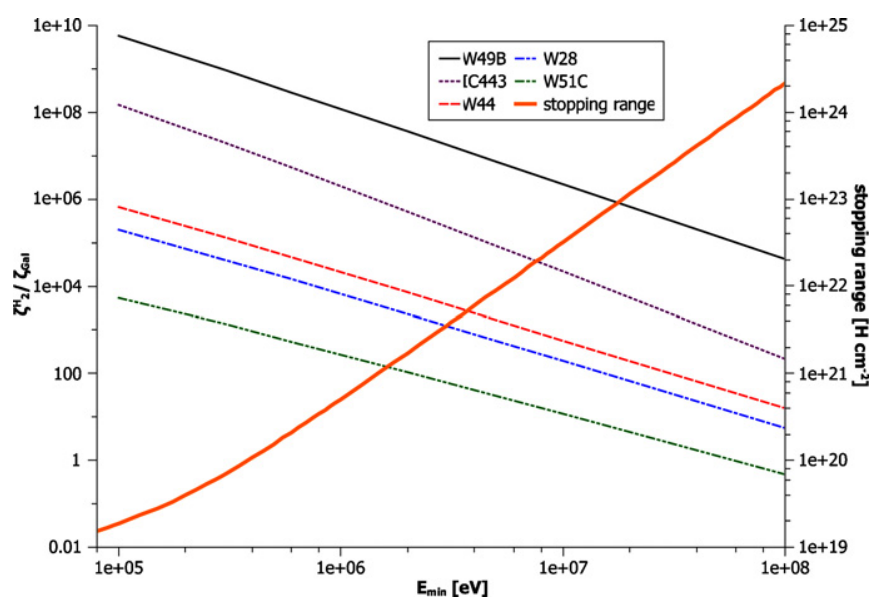

Figure 1. Ionization rate, normalized to $\zeta_{\mathrm{Gal}}:=2 \times 10^{-16} \mathrm{~s}^{-1}$ vs. $E_{\min }$. (A color version of this figure is available in the online journal.)

The escape of CRs from their sources and their propagation into surrounding material are also affected in complicated ways by interaction with magnetic fields (see Padovani \& Galli 2011 for a recent discussion for dense molecular gas). These issues will be addressed in future work.

\section{COSMIC-RAY-INDUCED MOLECULAR IONS}

As shown below, the observable line intensities of molecular ions spawned by $\mathrm{CR}$ ionization depend upon the ionization rate integrated through a column of molecular gas

$$
\eta=\int_{0}^{L} \zeta^{\mathrm{H}_{2}} n\left(\mathrm{H}_{2}\right) d \ell \approx \zeta^{\mathrm{H}_{2}} n\left(\mathrm{H}_{2}\right) L \mathrm{~s}^{-1} \mathrm{~cm}^{-2},
$$

which we call the cosmic ray exposure. The number density of interstellar $\mathrm{H}_{2}$ is $n\left(\mathrm{H}_{2}\right)$ and the integration is over path length $\ell=0$ to $L$. Because the CRs of shortest range die off at small $\ell$, protons at energies significantly greater than $E_{\min }$ contribute to the exposure. In principle, the exposure should be computed through an explicit depth integration of the discrete energy loss. The SNRs discussed in this context have a CR exposure of the order of $\eta \sim 10^{9} \mathrm{~s}^{-1} \mathrm{~cm}^{-2}$ or higher (see Figure 1), which is $>10^{4}$ times larger than the exposures that characterize diffuse clouds in the Galactic disk. In general, ionization by X-rays can produce similar chemical signatures. We expect the X-rays from SNRs to be much less important than CRs because the X-ray luminosities are relatively small and their depths of penetration are too short to yield high values of the exposure.

The outlines of interstellar ion chemistry have been understood for almost $40 \mathrm{yr}$ (Herbst \& Klemperer 1973) and the rate coefficients of all the crucial reactions are well determined. In steady state between the rates of source and sink reactions, the number densities of the transient ions $\mathrm{O}^{+}, \mathrm{OH}^{+}, \mathrm{H}_{2} \mathrm{O}^{+}$, and $\mathrm{HeH}^{+}$ are expected to be proportional to $\zeta_{\mathrm{H}}$. In fully molecular regions, $n(\mathrm{H}) \ll n\left(\mathrm{H}_{2}\right)$, of low ionization $x\left(e^{-}\right)<10^{-5}$, competing processes rarely interrupt the sequence of $\mathrm{H}$-atom abstraction reactions so that almost every ionization of hydrogen leads to formation of $\mathrm{H}_{2}^{+}, \mathrm{H}_{3}^{+}, \mathrm{OH}^{+}, \mathrm{H}_{2} \mathrm{O}^{+}$, and $\mathrm{H}_{3} \mathrm{O}^{+}$. The timescales to achieve steady state are very short, $\sim 1\left(1000 \mathrm{~cm}^{-3} / n\left(\mathrm{H}_{2}\right)\right)$ yr.

The abundances of interstellar molecules can be computed from a system of rate equations for various values of the physical conditions (density, temperature, ionization rate) and gas-phase elemental abundances, either in the steady-state limit or as a time-dependent solution. However, the abundances alone do not characterize observable signatures, because the distributions of the molecules among their quantum states tend to be far out of equilibrium. The departures from equilibrium will be most extreme for the most reactive molecular ions, because elastic and inelastic collisions that would otherwise thermalize them are typically not faster than the reactive collisions with the main collision partner, $\mathrm{H}_{2}$, that destroy them (Black 1998). We illustrate the coupled effects of ion chemistry and nonequilibrium excitation with results from a reference model of molecular gas at density $n\left(\mathrm{H}_{2}\right)=100 \mathrm{~cm}^{-3}$ exposed to an ionization rate $\zeta^{\mathrm{H}_{2}}=10^{-12} \mathrm{~s}^{-1}$ over a path length $L=10^{19} \mathrm{~cm}$ $=3.24$ pc. The excitation and transport of spectrum lines are computed through use of the Radex program, which can incorporate formation and destruction rates state-by-state for reactive molecules along with all relevant radiative and inelastic collision processes (van der Tak et al. 2007). The kinetic temperature of the molecular gas is assumed to be $150 \mathrm{~K}$, somewhat elevated owing to enhanced heating by CRs. All line widths are taken to be $9.394 \mathrm{~km} \mathrm{~s}^{-1}$. Full details of these calculations will be presented elsewhere. Because $\mathrm{H}_{2}^{+}$has no permanent dipole moment, its vibration-rotation and pure rotational spectrum occurs only as weak electric quadrupole transitions. Upon birth by ionization of $\mathrm{H}_{2}, \mathrm{H}_{2}^{+}$is endowed with high vibrational excitation but it retains the relatively cold rotational distribution of its parent. At gas densities below $10^{4} \mathrm{~cm}^{-3}$, excited $\mathrm{H}_{2}^{+}$decays to the ground state by radiation more rapidly than it is destroyed or excited by collisions. As a result, the emitted spectrum in our reference model is directly predictable from the value of the CR exposure, $\eta=10^{9}$ $\mathrm{s}^{-1} \mathrm{~cm}^{-2}$, and the known quadrupole transition probabilities (Posen et al. 1983). Owing to symmetry breaking in the last few bound vibration-rotation levels near the dissociation limit, $\mathrm{H}_{2}^{+}$exhibits a few dipole-allowed radio-frequency transitions (Brown \& Carrington 2003; Howells \& Kennedy 1991; Moss 1993; Bunker 2000; Critchley et al. 2001). These transitions are excited in our model by the small fraction of ionizations that put $\mathrm{H}_{2}^{+}$into levels $(v=19, J=0,1)$ of the $1 s \sigma_{g}$ ground electronic state. Transitions to three barely bound levels ( $v=$ $0, J=0,1,2)$ of the $2 p \sigma_{u}$ state occur with high probabilities (Moss 1993) that partly compensate for the low populations of the initial states: we predict that these transitions at 17.6, 52.9, and $96.4 \mathrm{GHz}$ will appear as weak masers in regions of high CR exposure. The electronically excited levels also radiate rapidly to $1 s \sigma_{g}(v=17-18, J=0-3)$ in transitions at millimeter/ submillimeter wavelengths. Although these symmetry-breaking transitions will probably be undetectably weak in sources like those considered here, they may become observable in the more extreme environments in active galactic nuclei.

The high excitation of $\mathrm{H}_{2}^{+}$is partly transferred to $\mathrm{H}_{3}^{+}$in the next chemical step and $\mathrm{H}_{3}^{+}$can also be excited by collisions several times during its lifetime. The distortion-induced rotational transition (Pan \& Oka 1986) of $\mathrm{H}_{3}^{+}(J, K)=(4,4) \rightarrow(3,1)$ easily suffers population inversion (Black 1998), a necessary condition for a maser. The transition frequency is poorly known (approximately $217.7 \mathrm{GHz}$ ), but the line is predicted in our reference model to appear as a weak maser with a peak Rayleigh-Jeans brightness temperature of $10 \mathrm{mK}$, an intensity that is detectable with existing radio telescopes. The intrinsically weak, far-infrared rotational transitions of $\mathrm{H}_{3}^{+}$are excited mainly by collisions at the kinetic temperature of the gas, while the infrared vibration-rotation transitions of both $\mathrm{H}_{2}^{+}$and $\mathrm{H}_{3}^{+}$are excited in the formation process with superthermal intensity distributions. In general, the rotational line intensities of $\mathrm{H}_{3}^{+}$ 

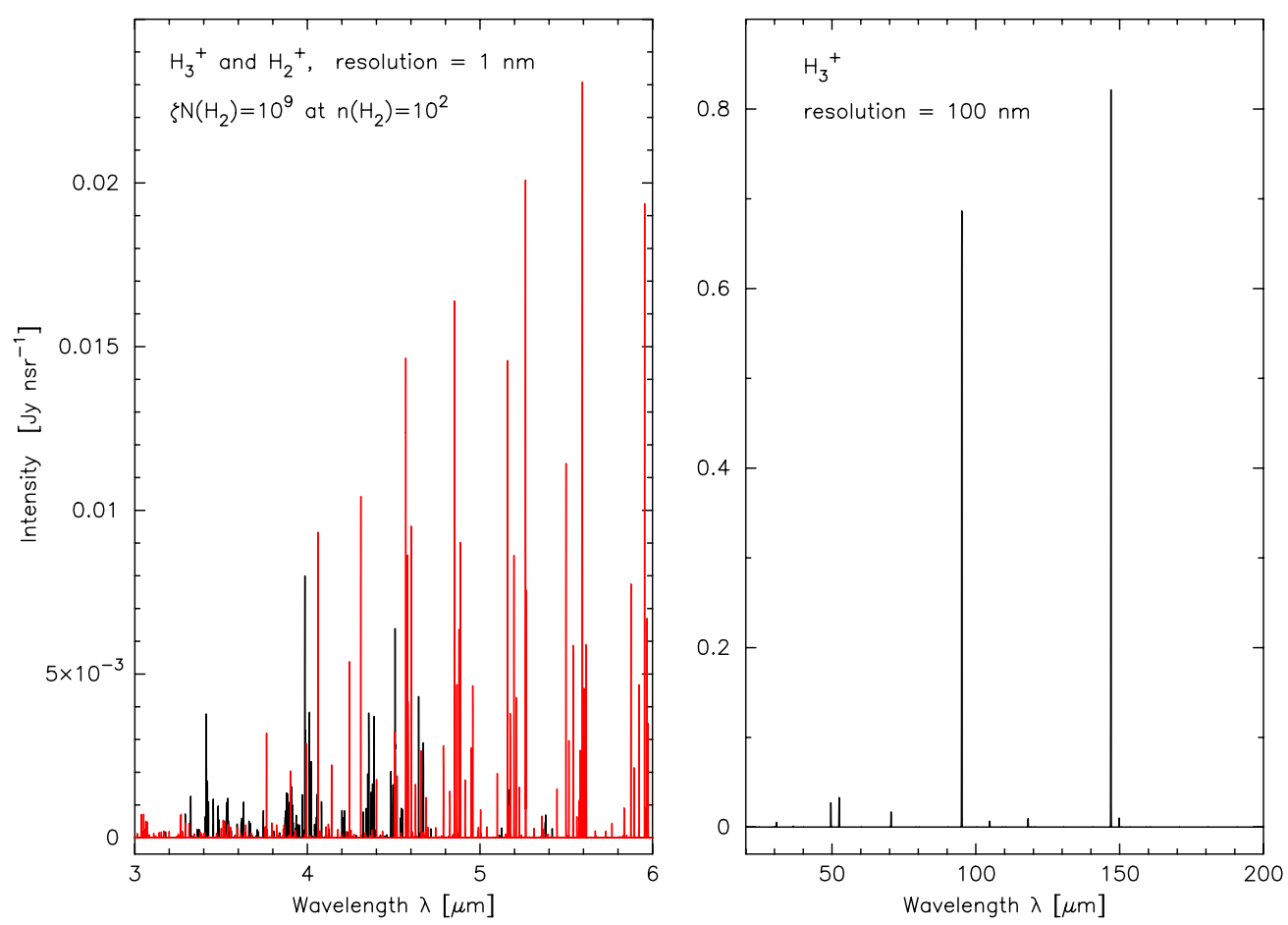

Figure 2. Predicted emission spectra of $\mathrm{H}_{2}^{+}$(red in the online version) and $\mathrm{H}_{3}^{+}$(black in the online version) for the reference model. The surface brightness is displayed in $\mathrm{Jy} \mathrm{nsr}^{-1}$, where $\mathrm{Jy}=10^{-26} \mathrm{~W} \mathrm{~m}^{-2} \mathrm{~Hz}^{-1}$ and $\mathrm{nsr}=10^{-9}$ steradian of solid angle. Here, $\eta=10^{9} \mathrm{~s}^{-1} \mathrm{~cm}^{-2}$ is used as a typical value in this context (see the text). (A color version of this figure is available in the online journal.)

will depend upon the local density and temperature; however, the entire spectrum of $\mathrm{H}_{2}^{+}$is rather insensitive to these physical conditions. We calculate explicitly the formation rate of $\mathrm{HeH}^{+}$ by vibrationally excited $\mathrm{H}_{2}^{+}$and predict the spectrum that results from its initial, superthermal excitation. The predicted spectra are illustrated in Figure 2. At infrared wavelengths the strongest lines of $\mathrm{H}_{3}^{+}$appear between 3 and $4.6 \mu \mathrm{m}$ while $\mathrm{H}_{2}^{+}$lines of comparable intensity appear between 4 and $6 \mu \mathrm{m}$. The strongest vibration-rotation lines of $\mathrm{HeH}^{+}$in this region are several hundred times weaker. In the far-infrared region, the thermally excited lines of $\mathrm{H}_{3}^{+}$are quite strong while the lines of $\mathrm{HeH}^{+}$are again considerably weaker. In the far-infrared, thermal emission by interstellar dust particles will produce continuous radiation with typical surface brightness $\geqslant 0.1 \mathrm{Jy} \mathrm{nsr}^{-1}$ at a wavelength of $100 \mu \mathrm{m}$; even so, it appears that the strongest rotational lines of $\mathrm{H}_{3}^{+}$might be observable if the spectroscopic resolving power is at least 100 (resolution of $1 \mu \mathrm{m}$ at $100 \mu \mathrm{m}$ wavelength). For comparison, the weak $\mathrm{H}_{3}^{+}$feature at $30.725 \mu \mathrm{m}$ has an intensity approximately $1 / 2$ as large as that of the $\mathrm{H}_{2} J=2 \rightarrow 0$ line at $28.226 \mu \mathrm{m}$. We have also calculated the spectra of the $\mathrm{OH}_{n}^{+}$ ions, $n=1-3$. These ions form mainly as a result of reactions of thermalized $\mathrm{H}_{3}^{+}$or $\mathrm{H}^{+}$with oxygen and subsequent reactions with $\mathrm{H}_{2}$. The excess energy (enthalpies of reactants minus products) of each reaction will go partly into vibrational and rotational excitation of the product, so that the $\mathrm{OH}_{n}^{+}$ions will also exhibit superthermal excitation. A predicted signal of enhanced production of oxygen-bearing ions will be strong absorption in rotation-inversion transitions arising in highly metastable states of $\mathrm{H}_{3} \mathrm{O}^{+}$at frequencies 1.6-1.9 THz. Absorption spectroscopy of interstellar $\mathrm{H}_{3}^{+}$has already suggested enhanced rates of ionization throughout the central molecular zone of the Galaxy (Goto et al. 2008; Geballe \& Oka 2010) and in two locations near the SNR IC 443 (Indriolo et al. 2010). We predict that emission lines of both $\mathrm{H}_{2}^{+}$and $\mathrm{H}_{3}^{+}$will be detectable and that their intensities can be used to measure the CR exposure.

\section{SUMMARY}

Based on the hadronic interpretation of photon emission seen toward SNRs, we have calculated the associated CR ionization rate in SNR-MC systems. While the exact value will depend on the exact density and spectral shape, it is generally expected to be significantly above the Galactic average. The CR exposure of the interstellar hydrogen (ionization rate times gas density integrated over path length) for the SNRs W51C, W28, W44, IC443, and W49B is expected to be of the order of $\eta=10^{9} \mathrm{~s}^{-1} \mathrm{~cm}^{-2}$. This permits the construction of a coupled model of the ion chemistry and the excitation of reactive molecular ions that might serve as specific tracers of CRs near their sources. Predictions are made of the intensities of rotational and vibration-rotation transitions of the reactive ions. This includes the first quantitative prediction of an astronomical spectrum of $\mathrm{H}_{2}^{+}$. It appears that superthermally excited emission lines of $\mathrm{H}_{2}^{+}$and $\mathrm{H}_{3}^{+}$may be detectable at wavelengths between 3 and $6 \mu \mathrm{m}$. Strong thermal emission of $\mathrm{H}_{3}^{+}$may be measurable in the far infrared at 50-150 $\mu \mathrm{m}$ wavelength. In particular, W49B seems to be an outstanding candidate for the detection, as the predicted ionization rate for the discussed parameter range is more than four orders of magnitude above the Galactic average. Future measurements by, e.g., Herschel and ALMA provide the opportunity to make observations of ionization signatures in SNR/MC systems and analyze their correlation to the gamma-ray emission. A recent observation suggests an enhanced excitation and abundance of ammonia toward W28 (Nicholas et al. 2011). Using the Radex code, it is possible to explain the observed spectrum as $\mathrm{CR}$ induced with an ionization rate of $4.5 \times 10^{-12} \mathrm{~s}^{-1}$ (a factor of $>10^{4}$ above the Galactic 
average). Further, $\mathrm{H}_{3}^{+}$absorption observed from the direction of IC443 indicates an enhanced ionization level of $2 \times 10^{-15} \mathrm{~s}^{-1}$ (a factor of 10 above the Galactic average; Indriolo et al. 2010). In the future, more observations must be performed to explicitly search for the expected signatures of $\mathrm{H}_{2}^{+}$and $\mathrm{H}_{3}^{+}$. Treating the propagation of the $\mathrm{CRs}$ in detail will allow us to give even more precise predictions of the expected emission regions.

We thank S. Casanova for helpful discussions, and acknowledge support from the Research Department of Plasmas with Complex Interactions (Bochum) and from the Lorentz Center, Leiden, The Netherlands. Research on interstellar chemistry at Chalmers has been supported by the Swedish Research Council and the Swedish National Space Board.

\section{REFERENCES}

Abdo, A. A., Ackermann, M., Ajello, M., et al. (Fermi Collaboration) 2009, ApJ, 706, L1

Abdo, A. A., Ackermann, M., Ajello, M., et al. (Fermi Collaboration) 2010a, ApJ, 718, 348

Abdo, A. A., Ackermann, M., Ajello, M., et al. (Fermi Collaboration) 2010b, Science, 327, 1103

Abdo, A. A., Ackermann, M., Ajello, M., et al. (Fermi Collaboration) 2010c, ApJ, 722, 1303

Abdo, A. A., Ackermann, M., Ajello, M., et al. (Fermi Collaboration) 2010d, ApJ, 712, 459

Becker, J. K. 2008, Phys. Rep., 458, 173

Benz, A. O., Bruderer, S., van Dishoeck, E. F., et al. 2010, A\&A, 521, L35

Biermann, P. L., Becker, J. K., Meli, A., et al. 2009, Phys. Rev. Lett., 103, 061101

Black, J. H. 1998, Faraday Discuss. R. Soc. Chem., 109, 257

Black, J. H. 2007, in Molecules in Space and Laboratory, ed. J. L. Lemaire \& F. Combes (Paris: S. Diana), 90

Black, J. H., \& Fazio, G. G. 1973, ApJ, 185, L7

Blasi, P., Gabici, S., \& Vannoni, G. 2005, MNRAS, 361, 907
Brown, J. M., \& Carrington, A. 2003, Rotational Spectroscopy of Diatomic Molecules (Cambridge: Cambridge Univ. Press)

Bruderer, S., Benz, A. O., van Dishoeck, E. F., et al. 2010, A\&A, 521, L44

Bunker, P. R. 2000, Chem. Phys. Lett., 316, 266

Critchley, A. D., Hughes, A. N., \& McNab, I. R. 2001, Phys. Rev. Lett., 86, 1725

Dalgarno, A., Yan, M., \& Liu, W. 1999, ApJS, 125, 237

Ellison, D. C., \& Bykov, A. M. 2011, ApJ, 731, 87

Geballe, T. R., \& Oka, T. 2010, ApJ, 709, L70

Gerin, M., de Luca, M., Black, J., et al. 2010, A\&A, 518, L110

Goto, M., Usuda, T., Nagata, T., et al. 2008, ApJ, 688, 306

Gupta, H., Rimmer, P., Pearson, J. C., et al. 2010, A\&A, 521, L47

Herbst, E., \& Klemperer, W. 1973, ApJ, 185, 505

Howells, M. H., \& Kennedy, R. A. 1991, Chem. Phys. Lett., 184, 521

Indriolo, N., Fields, B. D., \& McCall, B. J. 2009, ApJ, 694, 257

Indriolo, N., Blake, G. A., Goto, M., et al. 2010, ApJ, 724, 1357

Kamae, T., Karlsson, N., Mizuno, T., Abe, T., \& Koi, T. 2006, ApJ, 647, 692

Krełowski, J., Beletsky, Y., \& Galazutdinov, G. A. 2010, ApJ, 719, L20

Montmerle, T. 1979, ApJ, 231, 95

Montmerle, T. 2010, in ASP Conf. Ser. 422, High Energy Phenomena in Massive Stars, ed. J. Martí, P. L. Luque-Escamilla, \& J. A. Combi (San Francisco, CA: ASP), 85

Moss, R. E. 1993, Chem. Phys. Lett., 206, 83

Neufeld, D. A., Goicoechea, J. R., Sonnentrucker, P., et al. 2010, A\&A, 521, L10

Nicholas, B., Rowell, G., Burton, M. G., et al. 2011, MNRAS, 411, 1367

Ossenkopf, V., Müller, H. S. P., Lis, D. C., et al. 2010, A\&A, 518, L111

Padovani, M., \& Galli, D. 2011, A\&A, 530, A109

Padovani, M., Galli, D., \& Glassgold, A. E. 2009, A\&A, 501, 619

Pan, F., \& Oka, T. 1986, ApJ, 305, 518

Posen, A. G., Dalgarno, A., \& Peek, J. M. 1983, At. Data Nucl. Data Tables, 28,265

Schilke, P., Comito, C., Müller, H. S. P., et al. 2010, A\&A, 521, L11

Schlickeiser, R. 2002, Cosmic Ray Astrophysics (Berlin: Springer)

Strong, A. W., Moskalenko, I. V., \& Ptuskin, V. S. 2007, Annu. Rev. Nucl. Part. Syst., 57, 285

van der Tak, F. F. S., Black, J. H., Schöier, F. L., Jansen, D. J., \& van Dishoeck, E. F. $2007, A \& A, 468,627$

Wyrowski, F., van der Tak, F., Herpin, F., et al. 2010, A\&A, 521, L34

Zirakashvili, V. N., \& Ptuskin, V. S. 2008, ApJ, 678, 939 